\title{
On Facilitating RFID Tag Read Processes via a Simple Parameter Estimation
}

\author{
Young Jae Park ${ }^{*}$ Regular Member, Young Beom Kim ${ }^{* \circ}$ Lifelong Member
}

\begin{abstract}
In this paper, we first formulate an optimal design problem for RFID tag identification processes and then propose a simplified estimation method for determining optimal frame sizes and termination time under an independence assumption. Through computer simulations we show that the proposed scheme outperforms Vogt's scheme in terms of identification delay.
\end{abstract}

Key Words : RFID, Anti-collision protocols, Passive tags, DFSA

\section{I . Introduction}

Due to the ability to identify objects wirelessly without line-of-sight, RFID systems are becoming noticeably prevalent. RFID systems are rated to be particularly attractive for applications such as retail, inventory management, and supply-chain management [1], [2].

RFID systems consist of a reader and multiple tags. While the reader being powerful in terms of memory and computational resources, there are many types of tags depending on their computational

capabilities. Among various tag types, passive ones are becoming more and more popular for large scale deployments due to their low cost [2].

Collision due to simultaneous tag responses is one of the key issues in RFID systems. It results in wastage of bandwidth, energy, and increases identification delays. To minimize collisions, RFID readers must use an anti-collision protocol. The design of anti-collision protocols becomes more challenging on considering that the tags must be simple, cheap and small enough.

The anti-collision algorithm of RFID can be either deterministic or statistic. In this paper, we analyze Framed Slotted Aloha (FSA) based anti-collision protocols. Such protocols have the ability to adjust their frame size in accordance with varying tag population using a tag estimation function.

Consider a reader with $N$ tags in its interrogation zone (See Fig. 1 and Fig. 2 for conceptual diagrams). Initially, the reader starts the collision resolution process with an arbitrary frame size. Tags then choose a slot randomly to transmit their identification. The reader monitors the status of each slot and counts the number of slots filled with zero, one or multiple tag responses. This observation is then translated to a tag estimate, $\tilde{N}$ via a tag estimation function. Once an estimate is computed, the reader adjusts its frame size accordingly in such a way that the reading process can be terminated as soon as possible. In this vein, an inaccurate tag estimate can result in non-optimal frame sizes, which can again increase identification delay. Another factor that should be considered carefully in designing the reading process is to determine when to stop the reading process, namely termination time 


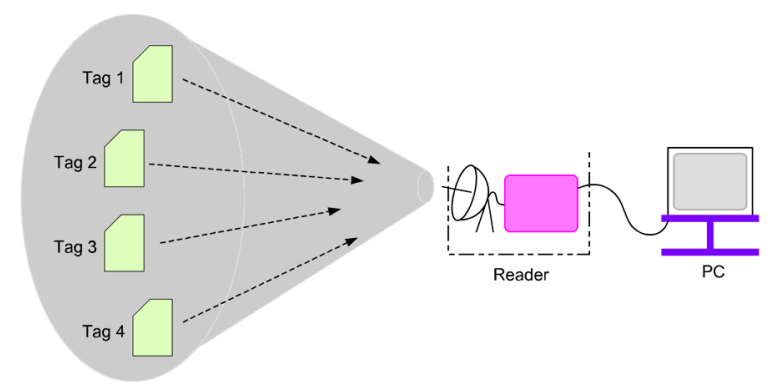

Figure 1. An example of RFID Systems.

$T_{\alpha}$. Here, $T_{\alpha}$ denotes the minimum number of read cycles required to identify all $N$ tags in the interrogation zone with probability $\alpha$, also referred to as assurance level.

In [3], [4], H. Vogt proposed a tag estimation function and a framework for choosing optimal frame sizes for computed tag estimates, including a procedure for adaptively reading a static set of tags. It is generally accepted that Vogt's tag estimation function based on Chebychev's inequality is the most accurate among several estimation functions [1]. However, though [3] provides a framework for determining the frame sizes and $T_{\alpha}$ suitable for a range of $N$ values, a closed form of solutions are not available and the actual computation of the two parameters therein is enormously burdensome and sometimes infeasible specially for large values of $N$.

In this paper, we first formulate an optimal design problem for RFID tag identification processes and then propose a simplified estimation method for determining optimal frame sizes and $T_{\alpha}$ under an independence assumption. In the proposed scheme, Young et al.'s estimate [5] is used instead of Vogt's estimate[3] for more secure estimation of $N$. Through computer simulations we show that the proposed scheme indeed outperforms Vogt's in terms of identification delay.

The remainder of this paper is organized as follows: We first formulate the optimal design problem for passive tag reading processes, including the introduction of Vogt's and Young et. al.'s schemes in the next section. In Section III, we propose a new scheme, including the derivation of

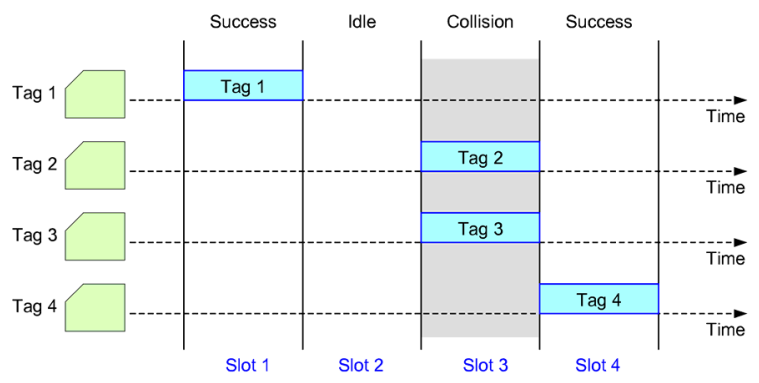

Figure 2. An sample outcome of DFSA read cycles.

some formulas useful for the estimation of optimal frame sizes and termination time. This is followed by a review of simulation results and discussion in Section IV. Section V concludes the paper.

\section{II . Preliminaries}

We consider RFID systems adopting passive tags and Dynamic Frame Slotted Aloha (DFSA) with no muting as the anti-collision protocol. DFSA can be defined as FSA protocols with variable frame sizes [2]. By DFSA with no muting, it is meant that no tags are informed of the outcome of each reading cycle from the reader. Similar to Basic Frame Slotted Aloha (BFSA), DFSA operates in multiple cycles but with the key difference that in each read cycle, the reader uses a tag estimation function to vary its frame size. A tag estimation function calculates the number of tags based on the information collected through each reading frame, consisting of the number of empty slots $(H)$, number of successful slots $(S)$, and number of collision slots $(C)$. This information is then used by the function to obtain a tag estimate, and hence the optimal frame size $L$ for a given cycle. Here, the optimal frame size is one which promises the minimum identification delay and maximum system efficiency including the lowest battery power consumption. Throughout this paper we assume the situation of static tag sets where the tags in the interrogation zone do not depart and no new tags arrive until the ongoing reading process is terminated by the reader.

\subsection{Problem Formulation}

In the previous section, Denote by $H_{t}, S_{t}, C_{t}$, 
respectively, number of empty slots, successful slots, collision slots, respectively, after $t^{\text {th }}$ read cycle. If we put $O_{t}=\left(H_{t}, S_{t}, C_{t}\right), t=1,2, \ldots$, then the vector $\left(O_{1}, O_{2}, \ldots, O_{t}\right)$ now represents the observation data collected until $t^{\text {th }}$ read cycle. With DFSA protocol in operation, the frame size $L$ is updated every read cycle. We denote by $L_{t}$, $t=1,2, \ldots$, the frame size used for the $t^{t h}$ read cycle. The value of $L_{1}$ is set to an appropriate value as a reading process starts (e.g., 16 [3]).

The optimal design problem for tag reading process can be formulated as follows:

Given an assurance level $\alpha$, for $t=1,2, \ldots$, devise tag estimate $N_{t}=f\left(O_{1}, O_{2}, \ldots, O_{t}\right)$, optimal frame size $L_{t+1}=g\left(N_{t}\right)$, and termination time $T_{\alpha}$

such that identification delay $\tau_{i d} \doteq \sum_{t=1}^{T_{\alpha}} L_{t}$ is minimized under the constraint that

$P\left[\right.$ all tags are identified after $T^{\text {th }}$ cycle $] \geq \alpha$.

Suppose there are $N$ tags in the interrogation zone (actually the number of tags $N$ is unknown to the reader) and we continue the read cycle with the same frame size $L$.

The question now is when to stop the reading process such that identification delay is minimized and with confidence level $\alpha$ all tags are read.

Table 1. Frame Sizes for Tag Estimates Suggested in [3]

\begin{tabular}{|c||c|c|c|c|c|}
\hline L & 16 & 32 & 64 & 128 & 256 \\
\hline \hline $\operatorname{Low}(\mathrm{N})$ & 1 & 10 & 17 & 51 & 112 \\
\hline $\operatorname{High}(\mathrm{N})$ & 9 & 27 & 56 & 129 & $\infty$ \\
\hline
\end{tabular}

\subsection{Vogt's Estimate [3]}

With $N$ tags in the interrogation zone and frame size $L$, the average number of slots with occupancy $r(r=0,1, \ldots, N)$, denoted by $a_{r}^{L, N}$ is given by

$$
a_{r}^{L, N}=L\left(\begin{array}{l}
N \\
r
\end{array}\right)\left(\begin{array}{l}
1 \\
L
\end{array}\right)^{r}\left(1-\frac{1}{L}\right)^{N-r}
$$

Based on the observations until $t^{t h}$ cycle, $\left\{\boldsymbol{O}_{1}, \boldsymbol{O}_{2}, \ldots, \boldsymbol{O}_{t}\right\}$, Vogt's estimate $E_{v d}$ is computed as $\epsilon_{v d}\left(\boldsymbol{O}_{t}\right)=\arg \min \left|\left(\begin{array}{c}a_{0}^{L_{t}, N} \\ a_{1}^{L_{t}, N} \\ a_{\geq 2}, N\end{array}\right)-\left(\begin{array}{c}H_{t} \\ S_{t} \\ C_{t}\end{array}\right)\right|$

where $\operatorname{argmin}_{N} f(N)$ denotes the value of $N$ maximizing $f(N)$ and $a_{\geq 2}^{L_{t}, N}$ denotes the average number of slots with occupancy greater than or equal to 2. Thus it always holds that $a_{0}^{L_{t}, N}+a_{1}^{L_{t}, N}+a_{\geq 2}^{L_{t}, N}=H_{t}+S_{t}+C_{t}=L_{t} . \quad$ It $\quad$ is noteworthy that Vogt's estimate utilizes only the last observation $\boldsymbol{O}_{t}$. Upon obtaining $N_{t}=\epsilon_{v d}\left(\boldsymbol{O}_{t}\right)$, the optimal frame size for the $(t+1)^{s t}$ cycle $L_{t+1}$ is determined according to Table I, supposed to yield lower identification delays for a given tag range. For example, when $N_{t}=12$, a frame size of 32 is considered optimal.

\subsection{Young et al.'s Estimate [5]}

A problem with Vogt's scheme is that the tag estimate does not converge to the actual value and continuously fluctuates as the reading process proceeds, due to the fact that Vogt's estimate utilizes only the most recent observation $O_{t}$ instead

of $O_{1}, O_{2}, \ldots, O_{t}$

In DFSA the tag estimate $N_{t}$ is computed after $t^{t h}$ cycle for $t=1,2, \ldots$ and the frame size $L_{t+1}$ for $(t+1)^{s t}$ read cycle is adjusted appropriately. As the tag reading process goes on, the frame size $L_{t}$ may or may not change. We can decompose the sequence of observations into multiple groups. Each group consists of consecutive observations with the same frame size is termed as a round [5]. In this vein, a read process can be organized as

$$
\begin{aligned}
& \underbrace{\boldsymbol{O}_{1}, \boldsymbol{O}_{2}, \ldots, \boldsymbol{O}_{n_{1}}}_{\text {round } 1}, \underbrace{\boldsymbol{O}_{n_{1}+1}, \boldsymbol{O}_{n_{1}+2}, \ldots, \boldsymbol{O}_{n_{1}+n_{2}}, \ldots}_{\text {round } 2} \\
& \underbrace{\boldsymbol{O}_{n_{1}+\ldots+n_{m-1}+2}, \ldots, \boldsymbol{O}_{n_{1}+\ldots+n_{m-1}+n_{m}}}_{n_{1}+\ldots+n_{m-1}+1}, \ldots .
\end{aligned}
$$




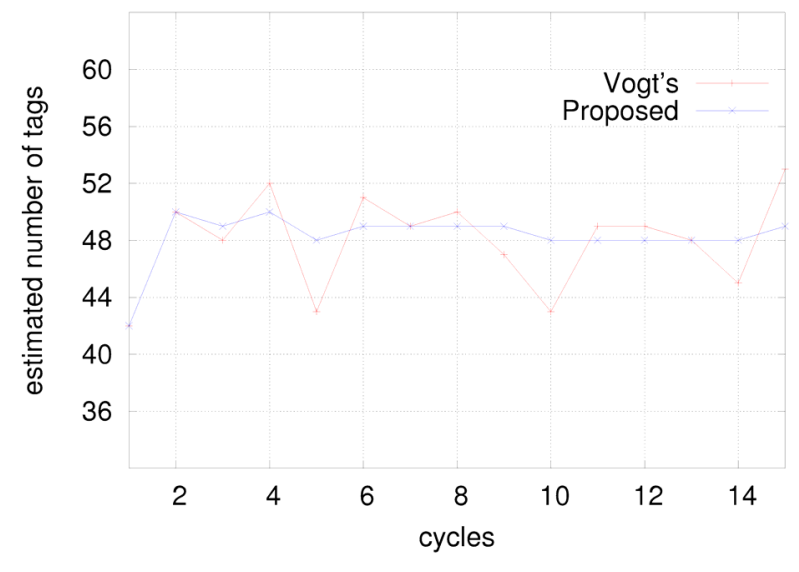

Figure 3. A sample trajectory of tag estimates when $\mathrm{N}=48$.

The sample averages after each read cycle in $m^{\text {th }}$ round $O_{n_{1}+\ldots+n_{m-1}+l}$ is given by

$O_{n_{1}+\ldots+n_{m-1}+l} \stackrel{\Delta}{=} \frac{1}{l} \sum_{s=1}^{l} O_{n_{1}+\ldots+n_{m-1}+s}, \quad l=1,2, \ldots$

Young et al.'s estimate $\epsilon_{y y}^{m, l}$ for $l^{\text {th }}$ cycle in $m^{\text {th }}$ round is given by

$$
\epsilon_{y y}^{m, l} \stackrel{\Delta}{=} \underset{N}{\arg \min }\left|\left(\begin{array}{c}
a_{0}^{L_{m}, N} \\
a_{1}^{L_{m}, N} \\
L_{m}, N
\end{array}\right)-O_{n_{1}+\ldots+n_{m-1}+l}\right| .
$$

Fig. 3 shows that the estimate $\epsilon_{y y}^{m, l}$ yields more stable results compared to $\epsilon_{v d}$.

\section{PROPOSED SCHEME}

Going back to the optimal design problem described in Section II, we can develop some formulas concerning suitable frame sizes and termination time $T_{\alpha}$. Suppose that tags are assigned serial numbers ranging from 1 to $N$. Let $E_{t}$ and $E_{t}^{i}$, respectively, denote the event that all tags are identified till the end of $t^{\text {th }}$ read cycle and the event that tag $i$ is identified till the end of $t^{t h}$ read cycle, respectively. Denoting by $P[E]$, the probability that an event $E$ will happen, it is straightforward to get

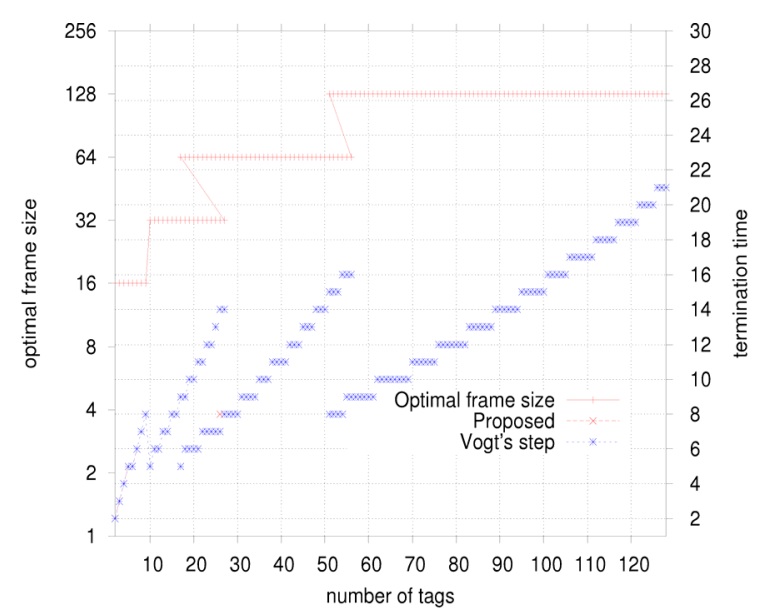

Figure 4. Comparison of Vogt's and proposed methods for computing optimal frame sizes.

$$
\boldsymbol{P}\left[E_{t}\right]=\boldsymbol{P}\left[\bigcap_{i=1}^{N} E_{t}^{i}\right]
$$

with

$$
P\left[E_{t}^{i}\right]=1-\left(1-\left(1-\frac{1}{L}\right)^{N-1}\right)^{t}
$$

If we assume that events $E_{t}^{1}, E_{t}^{2}, \ldots, E_{t}^{N}$ are independent (the assumption is not generally true), then we have

$$
P\left[E_{t}^{i}\right]=\left(1-\left(1-\left(1-\frac{1}{L}\right)^{N-1}\right)^{t}\right)^{N}
$$

With respect to the constraint (1), the termination time $T_{\alpha}$ can be determined as the minimum $t$ satisfying

$$
\left(1-\left(1-\left(1-\frac{1}{L}\right)^{N-1}\right)^{t}\right)^{N} \geq \alpha, \quad t=1,2, \ldots
$$

whence we have

$$
T_{\alpha}=\left\lceil\frac{\ln \left(1-\alpha^{1 / N}\right)}{\ln \left[1-\left(1-\frac{1}{L}\right)^{N-1}\right]}\right\rceil
$$

where the operator $\lceil x\rceil$ stands for the smallest integer greater than or equal to $x$. The exact value of $T_{\alpha}$ can be computed following the Markov chain approach described in [3]. However, the computation 


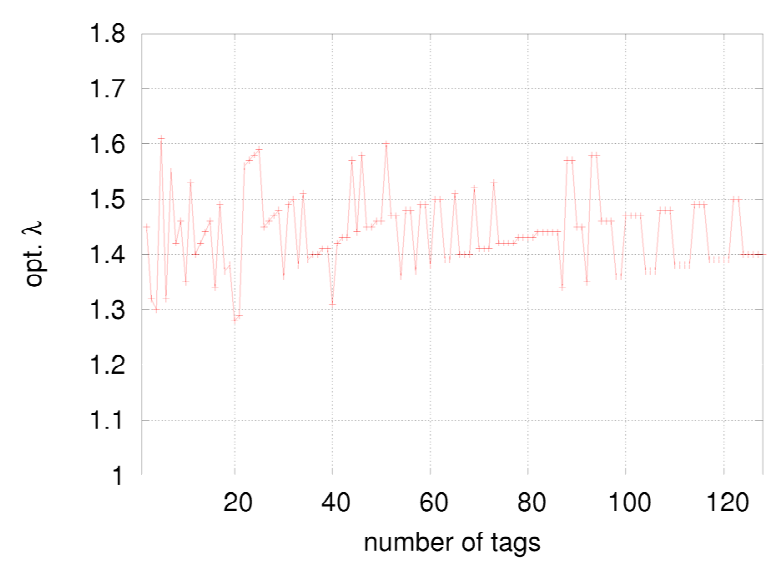

Figure 5. The values of $\lambda$ for optimal frame sizes.

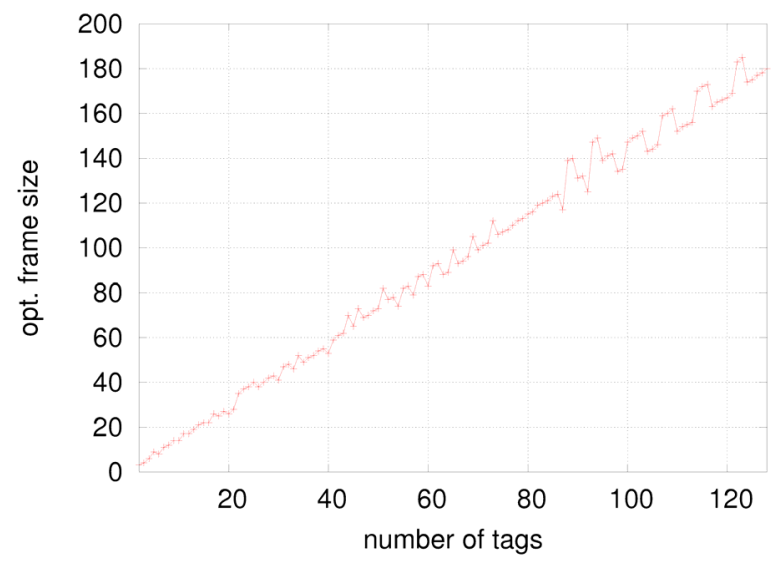

Figure 6. The optimal frame sizes vs. number of tags.

is extremely burdensome specially for large values of $N$. Though the independence assumption is not generally true, Fig. 4 shows that the values computed in both ways coincide exactly except only one case. Throughout we use the formula (2) for the estimation of $T_{\alpha}$.

This time we are interested in finding out the optimal frame size $L_{N}^{*}$ for given $N$, minimizing the identification delay $\tau_{i d}$, so that we have

$$
L_{N}^{*}=\underset{L=2,3, \ldots}{\arg \min } L\left\lceil\frac{\ln \left(1-\alpha^{1 / N}\right)}{\ln \left[1-\left(1-\frac{1}{L}\right)^{N-1}\right]}\right\rceil .
$$

By putting $L=\lambda N$ as was done before, $\tau_{i d}$ can be expressed as a function of $\lambda$, i.e.,

$\tau_{i d}=\lambda N\left\lceil\frac{\ln \left(1-\alpha^{1 / N}\right)}{\ln \left[1-\left(1-\frac{1}{\lambda N}\right)^{N-1}\right]}\right\rceil$

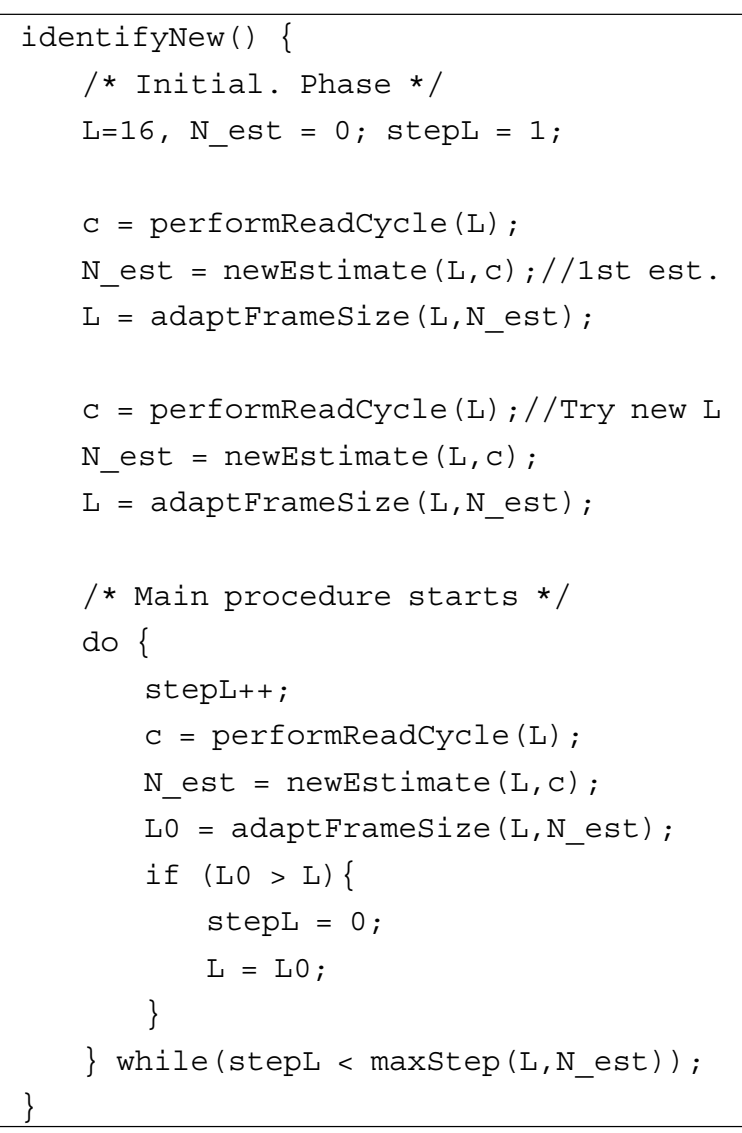

Figure 7. The tag read procedure used in the proposed scheme.

Fig. 5 shows that in most cases the optimal value of $\lambda$ falls in the interval $[1.3,1.6]$ and Fig. 6 also implies the tendency that the optimal frame size grows linearly in $N$. Thus, we can roughly compute the optimal frame size according to

$$
L_{N}^{*}=\lambda^{*} \times N \text { with } \lambda^{*} \in[1.3,1.6] .
$$

Finally, similar to the $\mathrm{Q}$ algorithm defined in ISO 18000-6 C[6] where only modest computational resources are required, we use the actual frame size $L_{N}^{\star}$ instead of $L_{N}^{*}$, given by

$$
L_{N}^{\star} \doteq 2^{\left\lceil\log _{2} L_{N}^{*}\right\rceil} .
$$

On the other hand, Vogt's procedure for reading tags [3] often generates overestimated values of $N$ and $L$, which lead to unnecessarily large values of $T_{\alpha}$, thereby resulting in greater identification delays. 


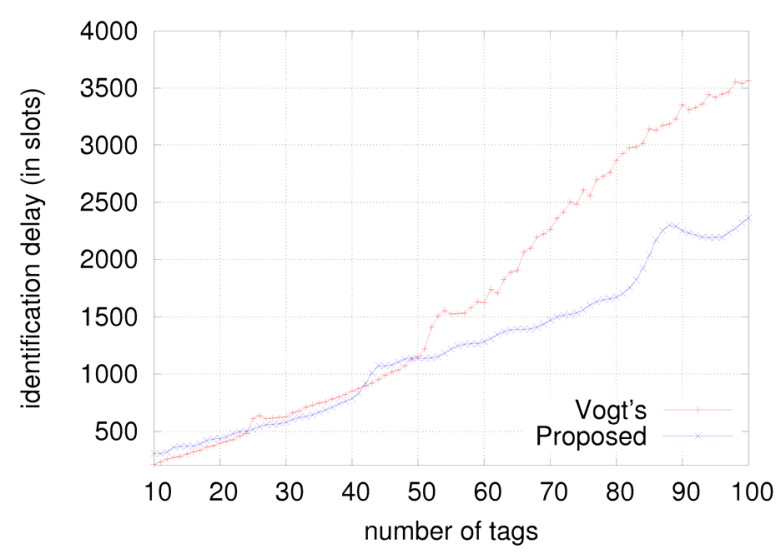

Figure 8. Performance of two schemes in terms of identification delay.

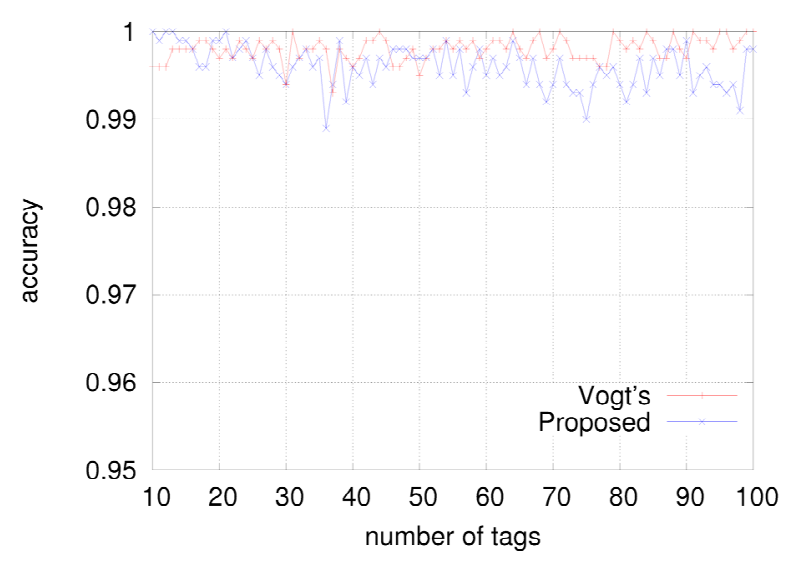

Figure 9. The identification accuracies of two schemes.

In order to circumvent this problem, we use the procedure described in Fig. 7. In this procedure, considering the cases of the initial value of $L$ being too small compared to $N$, the initial estimate for the number of tags is performed twice. In the first estimate, the value of $L$ is set to 16 while in the second estimate, the value of $L$ obtained in the first estimate is used for secure tag estimates.

\section{SIMULATION RESULTS AND DISCUSSION}

We performed computer simulations to evaluate the proposed scheme. The aspired assurance level $\alpha$ was set to 0.99 , meaning the requirement that on the average the number of cases failing to identify all tags should not exceed $1 \%$ of all

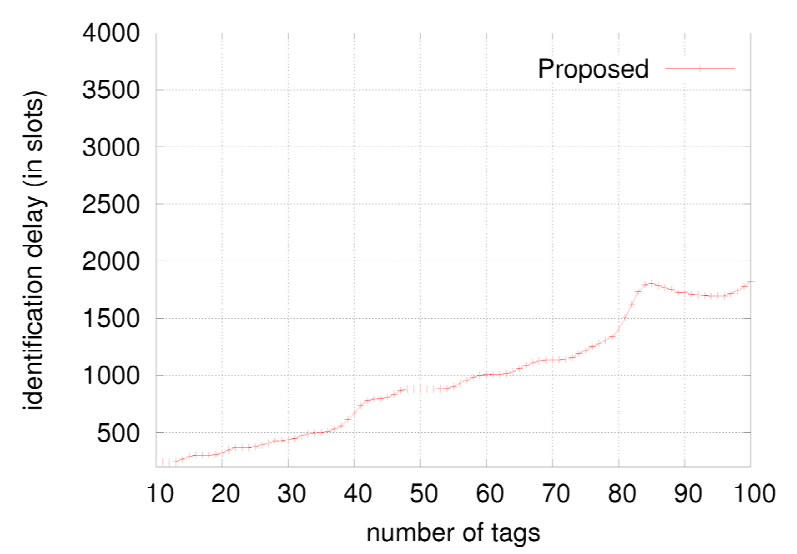

Figure 10. The identification delays of the proposed scheme for $\alpha=0.9$.

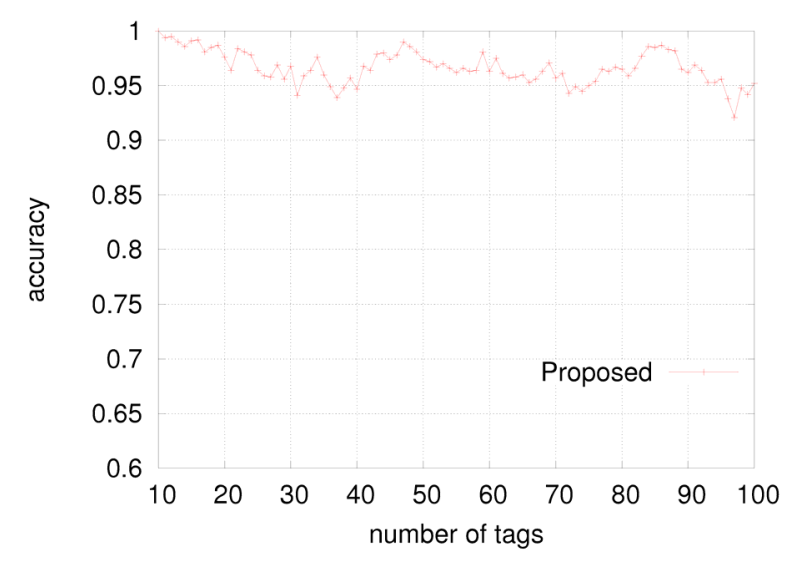

Figure 11. The identification accuracies of the proposed scheme for $\alpha=0.9$.

identification processes. In the simulations, we used the tag estimate $\epsilon_{y y}^{m, l}$ and the read procedure described in Fig. 7 while the optimal frame size was set in accordance with (3) and (5). The tag set size $N$ for the simulation ranges from 10 to 100 and for each tag set size we carried out 1000 runs of the reading procedure. Fig. 8 and Fig. 9 show that the proposed scheme works fairly well for the entire range of $N$ while the aspired accuracy level (i.e., 0.99) being satisfied all the time. For example, the identification delay for $N=80$ in the proposed scheme is shorter than that in Vogt's scheme by about 1,100 slots. Further, we can observe that the proposed scheme is more useful for large values of $N$. On the other hand, Fig. 9 shows that the actual accuracy of Vogt's scheme turns out to be unnecessarily far above the aspired accuracy level. Additionally, 
Fig. 10 and Fig. 11 show the performance of the proposed scheme for $\alpha=0.9$. Note in Fig. 8 that Vogt's scheme yields slightly better results for $N \in[10,25]$. This is due to the fact that in Vogt's read procedure $L$ is initially set to 16 , while having the overestimation problem for general values of $N$ as mentioned at the end of the previous section.

\section{CONCLUSION}

In this paper, we formulated an optimal design problem for RFID tag identification processes and proposed a new scheme via simple parameter estimation formulas for determining optimal frame sizes and $T_{\alpha}$ under an independence assumption, which are given in closed form and turn out to be very accurate. Through computer simulations we showed that the proposed scheme outperforms Vogt's scheme in terms of identification delay while satisfying the given assurance level.

\section{References}

[1] D. Klair, K.-W. Chin, and R. Raad, "On the Accuracy of Tag Estimation Functions, “ Sixth IEEE International Symposium on Communications and Information Technologies, Sydney, Australia (2007).

[2] D. Klair, K.-W. Chin, and R. Raad, "A Survey and Tutorial of RFID Anti-Collision Protocols," IEEE Communications Surveys \& Tutorials vol. 12, No.3 (2010), pp. 400-421.

[3] H. Vogt, "Efficient object identification with passive RFID tags," International Conference on Pervasive Computing (PerCom) (2002).

[4] H. Vogt, "Multiple object identification with passive RFID tags,' IEEE Intl. Conf. on Man and Cybernetics (2002), pp. 6-13.

[5] Y.B. $\sim$ Kim and Y.-J. Park, "On the performance enhancement of RFID anti-collision protocols using sample averages," to appear in the Proceedings of
IEEE Vehicular Technology Conference (2012).

[6] EPCglobal, Class 1 Generation 2 UHF Air Interface Protocol Standard Version 1.0.9, 2005.

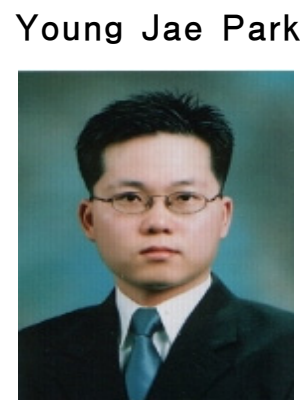

Regular Member received the B.S. and M.S. degrees in Electronics Engineering from Konkuk University, Seoul, Korea, in 2000 and 2006, respectively. Currently, he is pursuing his Ph.D. degree in the same university. His research interests include wireless mobile communication, ubiquitous, and next generation networks.

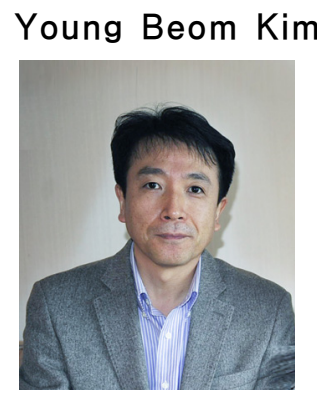

Lifelong Member received B.S. and M.S. degrees in electronics engineering from Seoul National University, Seoul, Korea in 1984 and 1986, respectively, and $\mathrm{Ph} . \mathrm{D}$. degree in electrical engineering from University of Maryland, College Park, USA, in 1996. From 1986 to 1988, he was with Korea Telecom, Seoul, Korea. He is currently with Konkuk University, Seoul, Korea. His research interests include traffic modeling and congestion control in high speed communication networks and wireless mobile communication. 